Acta Crystallographica Section D

Biological

Crystallography

ISSN 0907-4449

Editors: E. N. Baker and Z. Dauter

\title{
Using X-ray absorption spectra to monitor specific radiation damage to anomalously scattering atoms in macromolecular crystallography
}

V. Oliéric, E. Ennifar, A. Meents, M. Fleurant, C. Besnard, P. Pattison, M. Schiltz, C. Schulze-Briese and P. Dumas 
Acta Crystallographica Section D

Biological

Crystallography

ISSN 0907-4449

V. Oliéric, ${ }^{a}$ E. Ennifar, ${ }^{a}$

A. Meents, ${ }^{b}$ M. Fleurant, ${ }^{c}$

C. Besnard, ${ }^{\text {C }}$ P. Pattison, ${ }^{\text {,d }}$

M. Schiltz, ${ }^{c}$ C. Schulze-Briese ${ }^{b}$

and P. Dumas ${ }^{\mathrm{a} *}$

aIBMC-CNRS, UPR 9002, Université Louis Pasteur, F-67084 Strasbourg CEDEX, France, ${ }^{\mathbf{b}_{S}}$ wiss Light Source, PSI, CH-5232 Villigen, Switzerland, 'École Polytechnique Fédérale de Lausanne (EPFL), CH-1015 Lausanne,

Switzerland, and ${ }^{\mathbf{d}}$ Swiss-Norwegian Beamlines (SNBL), ESRF, F-38043 Grenoble CEDEX, France

Correspondence e-mail:

p.dumas@ibmc.u-strasbg.fr

\section{Using X-ray absorption spectra to monitor specific radiation damage to anomalously scattering atoms in macromolecular crystallography}

\begin{abstract}
Radiation damage in macromolecular crystals is not suppressed even at $90 \mathrm{~K}$. This is particularly true for covalent bonds involving an anomalous scatterer (such as bromine) at the 'peak wavelength'. It is shown that a series of absorption spectra recorded on a brominated RNA faithfully monitor the extent of cleavage. The continuous spectral changes during irradiation preserve an 'isosbestic point', each spectrum being a linear combination of 'zero' and 'infinite' dose spectra. This easily yields a good estimate of the partial occupancy of bromine at any intermediate dose. The considerable effect on the near-edge features in the spectra of the crystal orientation versus the beam polarization has also been examined and found to be in good agreement with a previous study. Any significant influence of the $(\mathrm{C}-\mathrm{Br}$ bond/beam polarization) angle on the cleavage kinetics of bromine was also searched for, but was not detected. These results will be useful for standard SAD/MAD experiments and for the emerging 'radiation-damage-induced phasing' method exploiting both the anomalous signal of an anomalous scatterer and the 'isomorphous' signal resulting from its cleavage.
\end{abstract}

\section{Introduction}

After the cooling of protein crystals to subzero temperatures was pioneered (Haas \& Rossmann, 1970; Douzou et al., 1975; Petsko, 1975) and the cryocrystallography technique was put into practice (Hope, 1988), it was hoped that crystals would become insensitive to radiation damage. However, from cryoelectron microscopy studies Henderson derived an estimate of around 20 MGy as the maximum X-ray dose that a crystal can receive without losing most of its diffraction power (Henderson, 1990). This prediction has been roughly verified (Gonzalez \& Nave, 1994; Teng \& Moffat, 2000). Recently, a better estimate of $30 \mathrm{MGy}$ was obtained after a detailed examination of the decrease of electron-density quality from several holoferritin and apoferritin crystals (Owen et al., 2006). It has now become clear that the extremely intense $\mathrm{X}$-ray beams at modern synchrotrons can significantly degrade diffraction even at $90 \mathrm{~K}$. Further to this now common observation, detailed studies were devoted to the more subtle radiation-induced effects in macromolecular crystals which take place before the occurrence of major damage. Almost simultaneous publications pointed out that protein crystals were not damaged randomly, but at specific sites, essentially disulfide bonds and the carboxylic groups of acidic residues (Burmeister, 2000; Ravelli \& McSweeney, 2000; Weik et al., 2000; for a recent review, see Ravelli \& Garman, 2006). It was also noticed that sites that were a priori equally susceptible
Received 12 March 2007 Accepted 19 April 2007 
were not affected identically. There are situations in which these observations can be explained in terms of significant differences in solvent accessibility and thus of exposure to the reactive radicals generated by $\mathrm{X}$-rays and secondary electrons (for example, Ramagopal et al., 2005). However, there are also examples of the opposite (Burmeister, 2000; Ravelli \& McSweeney, 2000; Fioravanti et al., 2007). Recently, the possible influence of local variations in the electric field on potentially damaging free electrons has been advocated (Holton, 2007).

Although radiation damage to crystals of nucleic acids has been less studied, it was noticed that bromine covalently attached to the $\mathrm{C} 5$ of uridine could be cleaved sufficiently during diffraction data collection, preventing phasing by the MAD method (Ennifar et al., 2002). It was suggested that this obviously negative observation might be turned into a positive one by considering the progressive cleavage of the anomalous scatterers as yielding SIR-like phase information. In this approach, the crystal with uncleaved bromine at the beginning of a data collection should be viewed as a 'heavy-atom derivative crystal' that is progressively transformed into a 'native crystal' during irradiation. For this procedure to be effective, it was noticed that it is necessary to keep the individual measurements of symmetry-related intensities unmerged in order to preserve the small differences resulting from the drift from full bromine occupancy to complete cleavage. In fact, it was originally anticipated by Ennifar et al. (2002) that the method would work better with iodine $(Z=53)$ instead of the lighter bromine $(Z=35)$ because of the larger SIR signal. This expectation was overly pessimistic, since it was subsequently shown that the phasing power was sufficient not only with bromine (Schiltz et al., 2004), but also with sulfur (Ravelli et al., 2003). Examples of successful phasing of protein crystal structures using radiation damage to iodine were reported by Evans et al. (2003) (after soaking the crystal in triiodide) and by Zwart et al. (2004) (after iodination of the tyrosines by chemical treatment). The method is now known under the acronym 'RIP' for 'radiation-induced phasing'. In the original implementation of the RIP method (Ravelli et al., 2003), two distinct data collections were separated by a step of deliberate irradiation of the crystal (without collecting data) in order to induce sulfur or bromine bond cleavage, whereas in Schiltz et al. (2004) the data were obtained after a single prolonged data collection (Ennifar et al., 2002) and the phasing software SHARP was modified to handle the dose-dependent bromine occupancy. The latter method is likely to be more efficient for the RIP method since the irradiation step necessary for cleaving the anomalous scatterers is also used for collecting useful diffraction data. Since then, the RIP method has also been used to exploit site-specific radiation damage to selenomethionines (Ravelli et al., 2005) and to a mercury derivative of a $60 \mathrm{kDa}$ protein (Ramagopal et al., 2005). It will certainly also be useful for iodo-derivatives, since the susceptibility of iodine to radiolysis is at least comparable to that of bromine or selenium at commonly used X-ray wavelengths (see, for example, Ennifar et al., 2003). Such iododerivatives are more commonly used with nucleic acids.
Furthermore, they can now be introduced into proteins as iodophenylalanine derivatives using the same techniques that are routinely used for incorporating selenomethionine (Xie $e t$ al., 2004). This extends the use of iodine as anomalous scatterer in proteins beyond the possibilities offered by triiodide soaking (Evans et al., 2003) and chemical iodination of tyrosines (Zwart et al., 2004).

It is thus clear that for proteins, as well as for nucleic acids, the RIP method has the potential to become a common phasing method by making use of the inevitable damage to sulfur, selenium, bromine and iodine. An initial goal of this study is to yield easily accessible experimental means for evaluating the anomalous-scatterer occupancy at each stage of data collection. This is particularly important because sitespecific radiation damage does not result in significant loss of the overall diffraction quality. In fact, in the above-mentioned case of a brominated RNA molecule the data-reduction statistics did not reveal anything unusual and only a detailed postmortem analysis, once the structure had been solved by other means, revealed that specific radiation damage at the $\mathrm{Br}$ sites had occurred (Ennifar et al., 2002). In the current context of high-throughput crystallography, it is essential to have a means for the early detection of specific radiation damage to the anomalous scatterers in the crystal, preferably during the $\mathrm{X}$-ray data collection, without removing the crystal from the diffractometer. In the present study, we show that the recording of X-ray absorption spectra at one of the edges of the anomalously scattering atoms can provide such a means. $\mathrm{X}$-ray absorption spectra are now routinely recorded (in fluorescence mode) at the start of a MAD experiment in order to make an optimal choice for the X-ray wavelengths that are selected for the subsequent collection of diffraction data. However, the information content of such spectra is much richer since the near-edge features (XANES) and the exact energy position of the absorption edge are functions of the chemical state of the absorbing atoms (Hodeau et al., 2001). Thus, when a $\mathrm{Br}$ atom that is initially bound covalently to a nucleotide is, most likely, reduced to a $\mathrm{Br}^{-}$ion by radiolysis, we can expect these chemical modifications to show up in the absorption spectrum at the $\mathrm{Br} K$ edge ${ }^{\mathbf{1}}$. Similarly, radiation damage at Se sites has been shown to introduce changes in the shape and energy position of the absorption-edge features (Holton, 2007). Also, it has been shown on model compounds that XANES spectra at the Se $K$ edge are sensitive to the oxidation state of the Se atom (Hunter et al., 1997). This was also very clear from crystals of photosystem II with an $\mathrm{Mn}_{4} \mathrm{Ca}$ complex that showed a variable oxidation state of manganese during irradiation (Yano et al., 2005).

We have also investigated the modulations in the absorption spectra that can be obtained by varying the crystal

\footnotetext{
${ }^{1}$ Alternatively to $\mathrm{Br}^{-}$, the cleavage product might be the radical $\mathrm{Br}$. However, the near-edge absorption spectrum of the latter is expected to exhibit a pronounced white-line feature corresponding to a resonant transition of the $1 \mathrm{~s}$ core electron to the vacant $4 \mathrm{p}$ valence state (for a discussion along these lines in the context of iodine, see Bressler et al., 2002). As described later, the spectra that we recorded after extensive irradiation did not display such a white line. Therefore, the final reaction product is most likely to be $\mathrm{Br}^{-}$.
} 
orientation. This phenomenon, which is a consequence of polarization anisotropy of X-ray absorption, has been known for a long time in small-molecule crystallography (for a comprehensive review, see Hodeau et al., 2001), but has only rarely been mentioned in the context of structural biology (Bricogne et al., 2005; Smith et al., 2001). Here, we provide an additional example of the importance of this purely geometrical effect that should neither be confused with radiation damage nor with changes in the oxidation state. We also checked whether such an orientation effect could explain the different cleavage kinetics for quite analogous covalent bonds in the same crystal.

When this paper was close to completion, two related studies appeared. The first described the monitoring of radiation damage by Raman spectra collected from crystals of a brominated DNA before and after irradiation to X-rays (McGeehan et al., 2007). The second, as in the present work, also made use of absorption spectra measurements to monitor radiation damages, but for $\mathrm{C}-\mathrm{Se}$ bonds and mostly on amorphous samples (Holton, 2007). Interesting comparisons will be made with these studies.

\section{Materials and methods}

\subsection{Crystals}

We have used crystals of a 23-mer RNA hairpin forming a so-called 'kissing complex' as a consequence of the selfcomplementarity within the loop sequence, which allows the formation of a dimer after loop-loop interaction (Ennifar et al., 2001). With a view towards using the MAD method, each monomer had been brominated by substituting a bromouridine for a uridine at position 3. These crystals will be referred to as DIS-BrU3 crystals in the following, DIS standing for 'dimerization initiation site' of HIV-1 genomic RNA. Two closely related types of DIS-BrU3 crystals were used in the present work, resulting from crystal availability when the experiments were performed. Both types belong to the orthorhombic space group $C 222_{1}$. The DIS-BrU3 crystals ( $a=27.1, b \simeq 115, c=95.3 \AA$ ) used at the Swiss Light Source (SLS, Villigen, Switzerland) were obtained using subtype $\mathrm{F}$ HIV-1 RNA (PDB code 2fd0). They were grown in complex with lividomycin (an aminoglycoside antibiotic) with methylpentanediol (MPD) as the precipitating agent, as described in Ennifar et al. (2006). The DIS-BrU3 crystals $(a=27.4$, $b \simeq 114.6, c=95.4 \AA)$ used at the European Synchrotron Radiation Facility (ESRF, Grenoble, France) were obtained in the absence of lividomycin with subtype B HIV-1 RNA (PDB code 1xpe). They were grown with PEG 8000 as the precipitating agent, as described elsewhere (Bernacchi et al., 2005; Ennifar \& Dumas, 2006). Both crystal types had a variable $b$ parameter from crystal to crystal. The two RNA sequences only differ by two G.C base pairs in the subtype B DIS being substituted by two A.U base pairs in the subtype F DIS. The two base pairs are located within the central helix of the kissing complex. It should be emphasized that the $\mathrm{C}-\mathrm{Br}$ covalent bonds susceptible to radiolysis are at a distance of $\sim 20 \AA$ from the closest variable base pair and of $\sim 15 \AA$ from the lividomycin molecule (in the subtype $\mathrm{F}$ crystals). As a consequence, neither the sequence variation nor the presence of the ligand is likely to have a direct influence on the $\mathrm{C}-\mathrm{Br}$ bond cleavage. The crystals grown under PEG-containing conditions were cryoprotected for $30 \mathrm{~min}$ in a solution containing 35\%(w/v) PEG 8000, 20\% MPD, $200 \mathrm{~m} M$ ammonium sulfate and $10 \mathrm{mM} \mathrm{MgCl}$. All crystals were flash-frozen in liquid ethane and maintained at $\sim 100 \mathrm{~K}$ in a nitrogen-gas stream delivered by an Oxford cryosystem during irradiation. The X-ray beam was defocused $(100 \times 150 \mu \mathrm{m}$ at the SLS and $400 \times 400 \mu \mathrm{m}$ at the ESRF) in order to ensure homogeneous irradiation of the entire volume of the crystals. The incidentbeam fluxes were obtained at the SLS from the photocurrent measured by a Si PIN diode (Syntef, Norway) on the beamline, assuming a linear relationship between the photocurrent and the flux at a given energy. For the ESRF, the PIN diode was calibrated at the Physikalisch-Technische Bundesanstalt (PTB) in Braunschweig (German Institute of Metrology).

\subsection{Recordings of absorption spectra and diffraction data collections at the SLS}

The measurements were made on beamline X10SA at the SLS. The photon flux without attenuation was $1.87 \times$ $10^{12}$ photons s $^{-1}$ at $13.4705 \mathrm{keV}$. Absorption spectra were collected in fluorescence mode using an energy sampling at $5 \mathrm{eV}$ steps below $13.460 \mathrm{keV}$ and above $13.489 \mathrm{keV}$ and at $1 \mathrm{eV}$ steps between these two energy values.

For the experiment involving only spectral recordings (see Fig. 1), the beam was attenuated variably to give a better dose sampling at the beginning of the experiment (successively $8 \%$, $30 \%$ and $100 \%$ of maximum intensity). For the experiment involving X-ray data collections intercalated between two consecutive recordings of spectra (see Figs. 2 and 3), 120 images were collected ( $1^{\circ}$ oscillation, $1 \mathrm{~s}$ exposure $)$ at the peak energy $(13.4732 \mathrm{keV}, \lambda=0.9202 \AA)$. In this case, all spectra were measured with the same beam attenuation $(20 \%$ of maximum intensity), whereas the 120 -image data collections were performed with variable beam attenuations $(17 \%$ of full intensity for data collections $1-4$ and $50 \%, 30 \%$ and $100 \%$ for data collections 5, 6 and 7, respectively). In Figs. 1(b) and 3, each cumulated dose representative of an experimental point for either a data collection or a spectrum included all previous doses plus half the dose received during that data collection or that spectrum (the other half being included in the cumulated dose of the next measurement).

\subsection{Recordings of polarized absorption spectra at the ESRF}

Polarized absorption spectra were collected on a single crystal of DIS-BrU3 at station BM01A of the SwissNorwegian Beamlines (SNBL) at the ESRF. The endstation features a KM6-CH six-circle kappa diffractometer manufactured by Oxford Diffraction Ltd, which allows orienting a crystal at will. Previous measurements have established that the degree of linear polarization of the delivered X-ray beam is better than 0.96 (Birkedal, 2000). A set of five diffraction 


\section{research papers}

frames were recorded to compute an orientation matrix. Polarized absorption spectra were measured in fluorescence mode with a Ce:YAP scintillation counter (Bede plc EDRc $\mathrm{X}$-ray detector) mounted in the horizontal plane and perpendicular to the incident-beam direction in order to minimize the background contribution from elastic scattering processes. For the recording of fluorescence spectra around the $\mathrm{Br} K$ edge (theoretical free-atom value $E=13474 \mathrm{eV}$, $\lambda=0.9202 \AA$ ), the following layout was used: (i) energy range 13 300-13 $440 \mathrm{eV}$ in steps of $2 \mathrm{eV}$, (ii) energy range 13440 13520 in steps of $0.5 \mathrm{eV}$, (iii) energy range 13 520-13 700 in steps of $2 \mathrm{eV}$ and (iv) energy range 13 700-13 800 in steps of $4 \mathrm{eV}$. The counting time at each step was $4 \mathrm{~s}$ and the incident beam was attenuated so as to obtain maximum counts of the order of $5 \times 10^{5}$. Absorption spectra were recorded for three different crystal orientations, in which the polarization direction of the X-ray beam was respectively aligned with the crystal directions $a, b$ and $c$. A total of 16 data sets, each consisting of three polarized absorption spectra, were recorded. In between measurements, the crystal was irradiated at $E=13700 \mathrm{eV}$ for variable amounts of time in order to induce debromination.

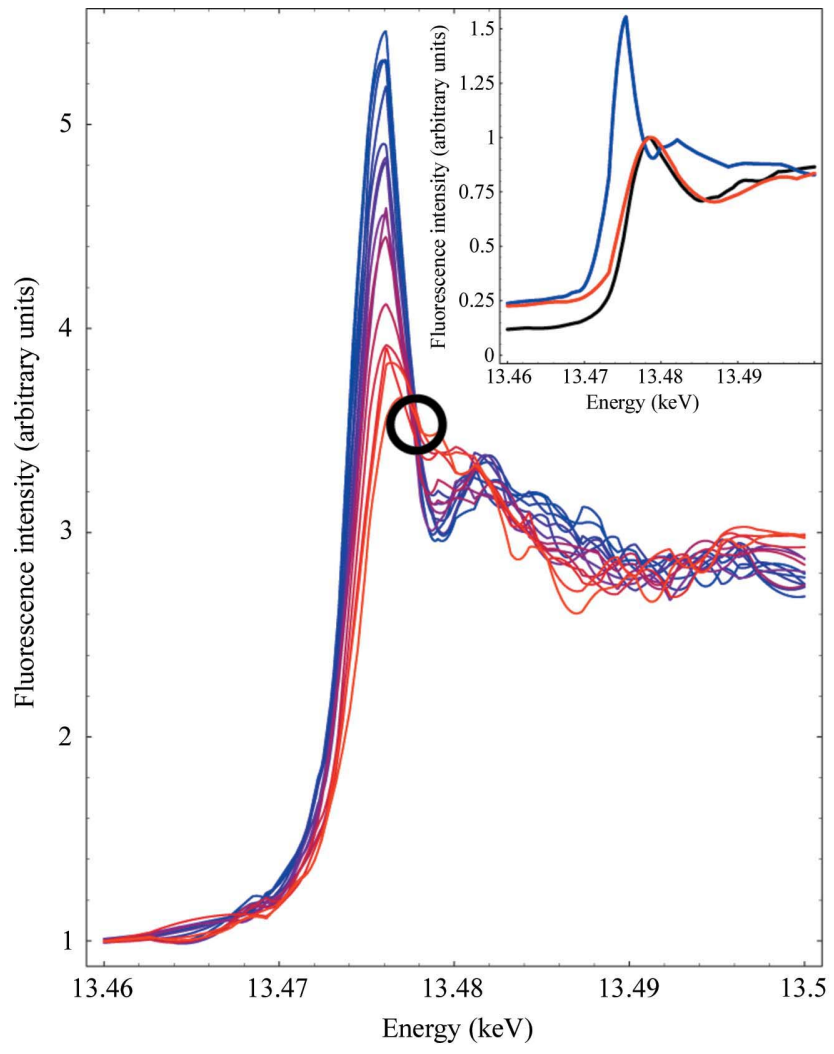

(a)

\subsection{Deriving occupancies from spectra}

To use (1) to derive the occupancy $\alpha_{i}$ corresponding to the $i$ th spectrum, a subset of relevant energy values between 13470 and $13490 \mathrm{eV}$ were used (see Fig. $1 b$ ). Because of the variable influence of noise, the exact energy limits were chosen case by case. Some improvement would be necessary for a fully automatic procedure. Each subset may be viewed as an $N$-component vector, $N$ being the number of experimental measurements in the selected energy window ( $N$ was close to 20). Straightforward calculations lead to the least-squares estimate $\alpha_{i}=\Delta \mathbf{F}_{i} \cdot \Delta \mathbf{F}_{0} / \Delta \mathbf{F}_{0} \cdot \Delta \mathbf{F}_{0}$, where $\Delta \mathbf{F}_{i}$ and $\Delta \mathbf{F}_{0}$ represent $N$-component vectors, respectively, for the differences $F_{i}(E)-F_{\infty}(E)$ and $F_{0}(E)-F_{\infty}(E)$ and the dot represents the usual scalar product.

\subsection{Calculations}

For the derivation of occupancies and Fig. 1, the absorption spectra were processed with specific programs developed using Mathematica 5.2 (Wolfram Research). The raw data were used to build interpolation functions consisting of second-degree polynomials between two successive points. For Fig. 4, the raw absorption spectra were converted to $f^{\prime}$ and $f^{\prime \prime}$ spectra using the program $\mathrm{CHOOCH}$ (Evans \& Pettifer, 2001). The diffraction data were processed using $D E N Z O$ and SCALEPACK (Otwinowski \& Minor, 1997). All other crys-

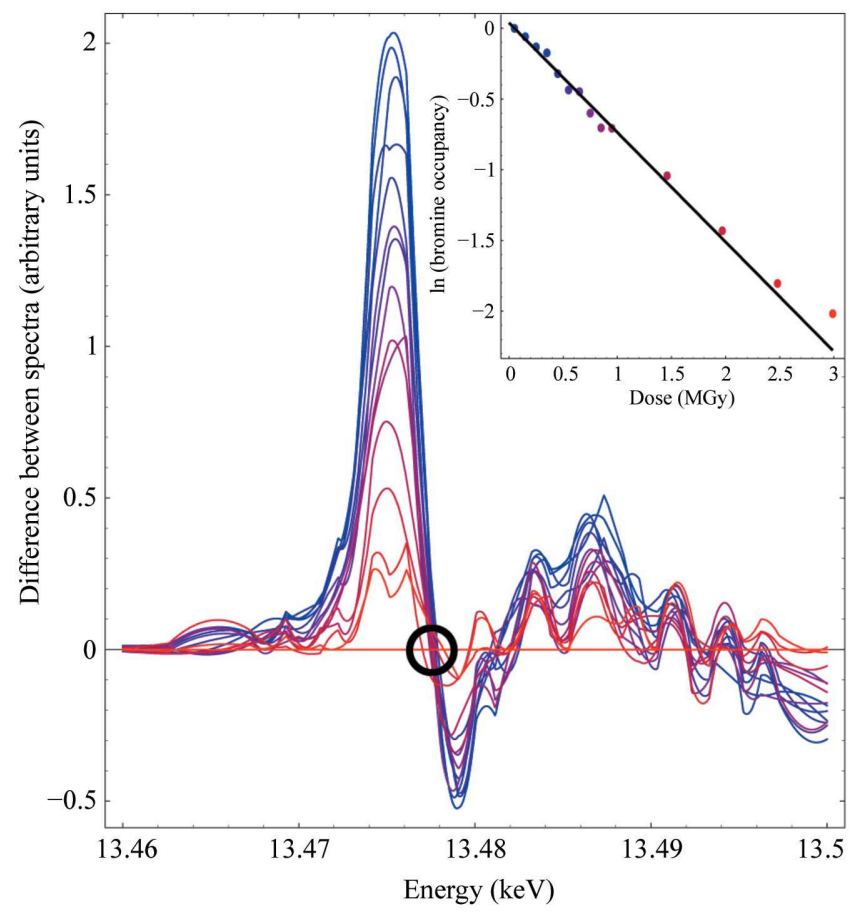

(b)

Figure 1

Dose-dependence of absorption spectra. (a) Evolution of 15 successive absorption spectra $F_{i}(E)$ recorded on one DIS-BrU3 crystal. The irradiation increases from blue ('zero dose') to red. The black circle marks the isosbestic point mentioned in the text. The insert shows a superimposition of an 'infinite-dose' spectrum $F_{\infty}(E)$ (red) and of the spectrum $F_{\mathrm{NaBr}}(E)$ obtained with $\mathrm{NaBr}$ in solution (black). The 'zero-dose' spectrum (blue) is shown for comparison. The red curve in the insert was obtained with another crystal that was more irradiated than that used for this experiment (the 15 th spectrum is still slightly displaced from the NaBr spectrum). (b) Superimposition of the difference spectra $F_{i}(E)-F_{15}(E) ; F_{15}(E)$ corresponds to the last spectrum, i.e. the closest to $F_{\infty}(E)=F_{\mathrm{NaBr}}(E)$. The insert shows the logarithm of bromine occupancy derived from (1) versus the cumulated dose. Strictly speaking, there exists a minor error in the occupancy values since $F_{15}(E)$ did not correspond exactly to $F_{\infty}(E)=F_{\mathrm{NaBr}}(E)$. The undulations appearing in $(b)$ are spurious features resulting from the interpolation. 
tallographic calculations were made with a specific package, BioCrystallographica (Ambert et al., 2006), which was also developed with Mathematica. The evaluation of doses was made with the program RADDOSE (Murray et al., 2004). For the doses corresponding to fluorescence spectra measurements, RADDOSE was used as many times as the number of sampling points in energy and the partial doses were added.

\section{Extracting the kinetics of anomalous scatterer cleavage from $X$-ray absorption spectra}

As reported previously, initial attempts to solve the structure using the MAD method failed owing to excessive bromine cleavage (Ennifar et al., 2002). In the present study, we have made two types of measurements. Firstly, we collected a sequence of absorption spectra in fluorescence mode, without diffraction data collection. In a second experiment, we inserted a diffraction data collection between each pair of consecutive spectra (see \$2). In the first case, therefore, the irradiation of the crystal resulted only from the absorption spectra. An example of such a series of spectra is shown in Fig. 1(a), where the colouring from blue to red corresponds to the sequence from the first to the last spectrum. This shows a continuous variation of the spectra with an important decrease of the intensity of the white line during irradiation [as obtained by Holton (2007) with selenium instead of bromine]. The variation is highlighted in Fig. 1(b), where the differences of each spectrum from the last spectrum have been superimposed. Importantly, the figures show an isosbestic point (black circles in Figs. $1 a$ and $1 b$ ). This is indicative of a 'twostate' situation in which each spectrum is merely a linear combination of two extreme spectra, that at 'zero dose' and that at 'infinite dose'. In other words, the $i$ th difference spectrum can be expressed as

$$
F_{i}(E)-F_{\infty}(E)=\alpha_{i}\left[F_{0}(E)-F_{\infty}(E)\right],
$$

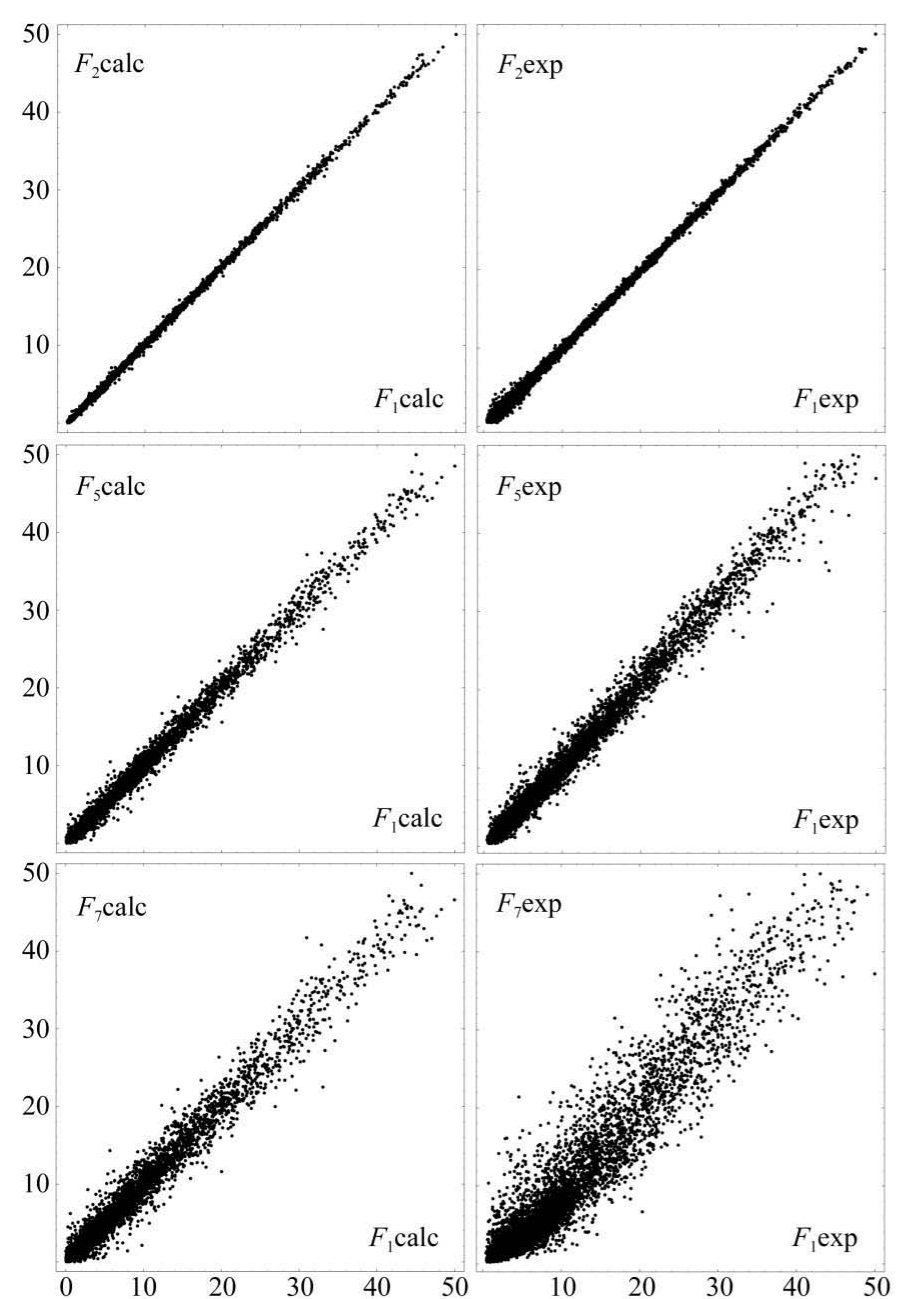

Figure 2

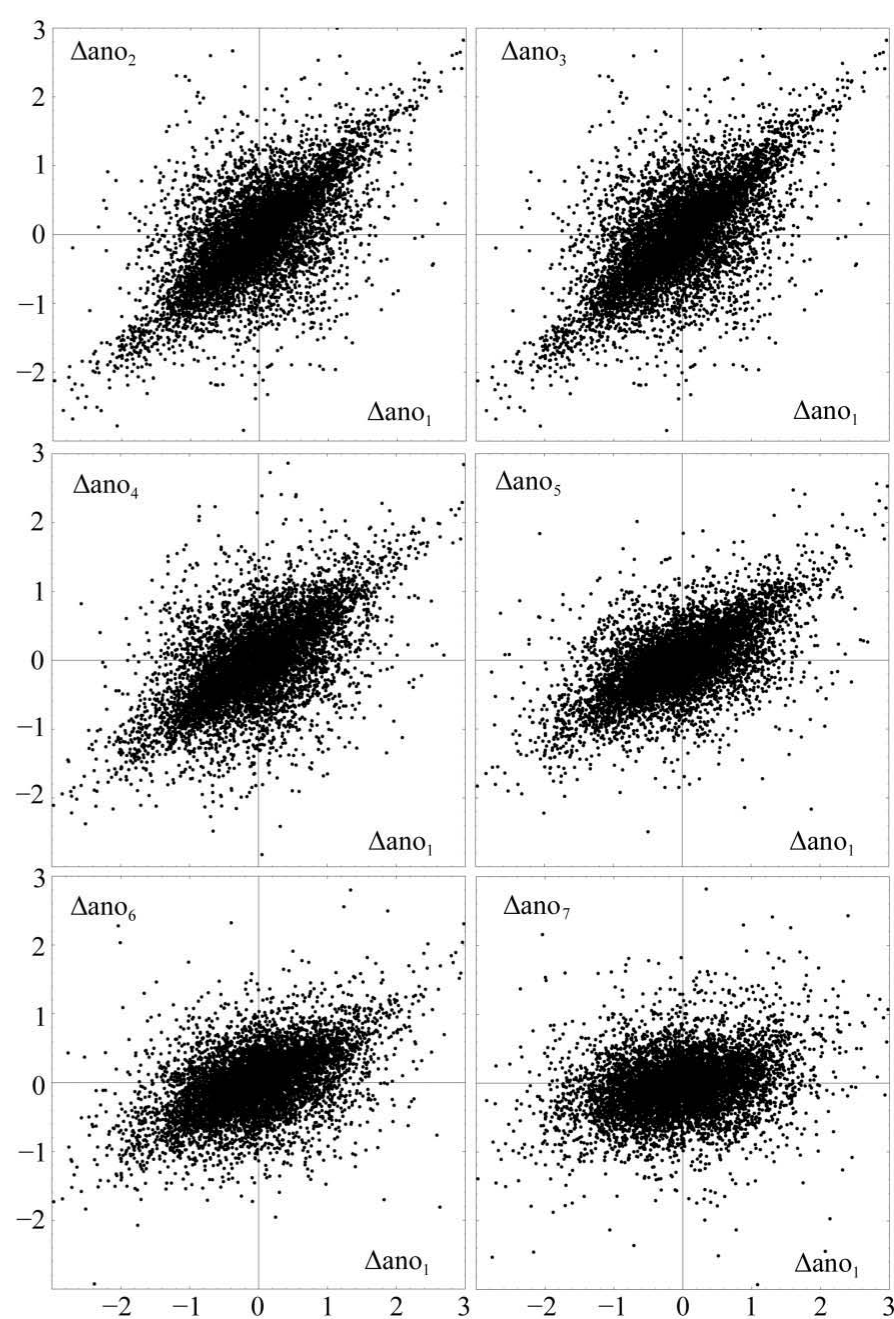

(b)

Cross-comparison of structure-factor amplitudes and anomalous differences. $(a)$ Pairwise comparison of experimental amplitudes $F_{1} / F_{i}$ (right-hand column) and of the corresponding calculated amplitudes (left-hand column) for 8645 common reflections in the resolution range $27.5-1.96 \AA$. The comparison is only shown for the pairs $F_{1} / F_{2}, F_{1} / F_{5}$ and $F_{1} / F_{7}$. The successive sets of calculated amplitudes were obtained using the following occupancy values of the two $\mathrm{Br}$ atoms in PDB entry $2 \mathrm{fd} 0$ : $0.96,0.96$ for $F_{1}, 0.49,0.58$ for $F_{5}$ and $0.15,0.24$ for $F_{7}$. These values were derived from the anomalous difference maps (see text and Fig. 3). The maximum $F$ values were arbitrarily scaled to 50. (b) Pairwise comparison of experimental anomalous differences $\Delta \mathrm{ano}_{1} / \Delta \mathrm{ano}_{i}$ for 7326 common acentric reflections. The $\Delta \mathrm{ano}_{i}$ values are consistent with the $F$ values in $(a)$. 


\section{research papers}

where $F_{0}(E)$ and $F_{\infty}(E)$ are ideal spectra at 'zero dose' and 'infinite dose', these being represented to a good approximation by the blue and red spectra, respectively, in Fig. 1( $a$ ). This situation arises because $\mathrm{Br}$ atoms contributing to the fluorescence signal are either covalently attached to the C5 of a uridine base or cleaved. An immediate requirement of such an interpretation is that $F_{\infty}(E)$, corresponding to full cleavage, should match the fluorescence spectrum of a solution of $\mathrm{NaBr}$, which was indeed verified (insert in Fig. 1a; see also Fig. 4). A practical consequence is that one can derive the extent of cleavage simply by scaling any intermediate difference spectrum $F_{i}(E)-F_{\infty}(E)$ to the maximum difference $F_{0}(E)-F_{\infty}(E)$ according to (1); the scaling factor $\alpha_{i}$ is nothing other than the fraction, i.e. the occupancy, of the not yet cleaved $\mathrm{Br}$ atoms. The results of such a derivation are shown in Fig. 1(b), where the logarithm of $\alpha_{i}$ has been plotted against the cumulated dose $d$ (insert in Fig. 1b). The linear variation indicates that the occupancy $Q$ varies as $Q(d)=\exp (-d / D), D$ being a refinable parameter, which is consistent with a first-order cleavage mechanism (or at least an apparent first-order mechanism). This is an independent confirmation of results obtained previously in connection with the RIP procedure (Ennifar et al., 2002; Schiltz et al., 2004) and more recently with selenium cleavage (Holton, 2007). From the linear fit in Fig. $1(b), D=1.33 \pm 0.03 \mathrm{MGy}$ was obtained. The low e.s.d. value of 0.03 MGy only reflects an accurate exponential dependence, but should not be viewed as representative of the accuracy of the absolute value of $D$, which is certainly poorer. The experiments performed to determine the effect of crystal orientation also involved deliberate irradiation steps (see Fig. 4), which allowed the derivation of $D=0.74$ $\pm 0.02 \mathrm{MGy}$. The same remark can be made on the low e.s.d. value.

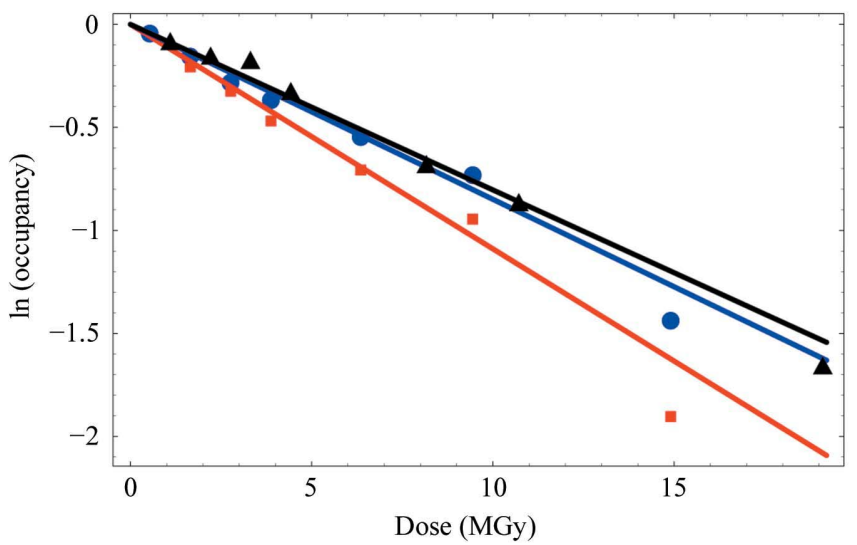

Figure 3

Comparison of bromine occupancies for the DIS-BrU3 crystals (black triangles) derived from fluorescence spectra variation as shown in Fig. 1 with those derived from anomalous difference maps (red squares and blue circles) corresponding to the data shown in Fig. 2(b). The red and blue colours correspond to the two $\mathrm{Br}$ atoms in the asymmetric unit. The difference between the two sites is little dependent on the resolution range used in the calculations. The more important departure from the blue and red regression lines at $15 \mathrm{MGy}$ arise from the large relative uncertainty of low occupancy values.
The two previous estimates $D=1.33 \mathrm{MGy}$ and $D=$ 0.74 MGy may be viewed as being in reasonable agreement considering that they were obtained on two different beamlines with related crystals obtained under different conditions with comparable, but not identical, molecules (see \$2). Recently, a Raman study on a DNA crystal that also contained two $\mathrm{Br}$ atoms in the asymmetric unit confirmed an exponential dependence (McGeehan et al., 2007). However, a much larger $D$ value was obtained (7.1 MGy). Such a large difference might be thought to originate from the unrelated crystals used

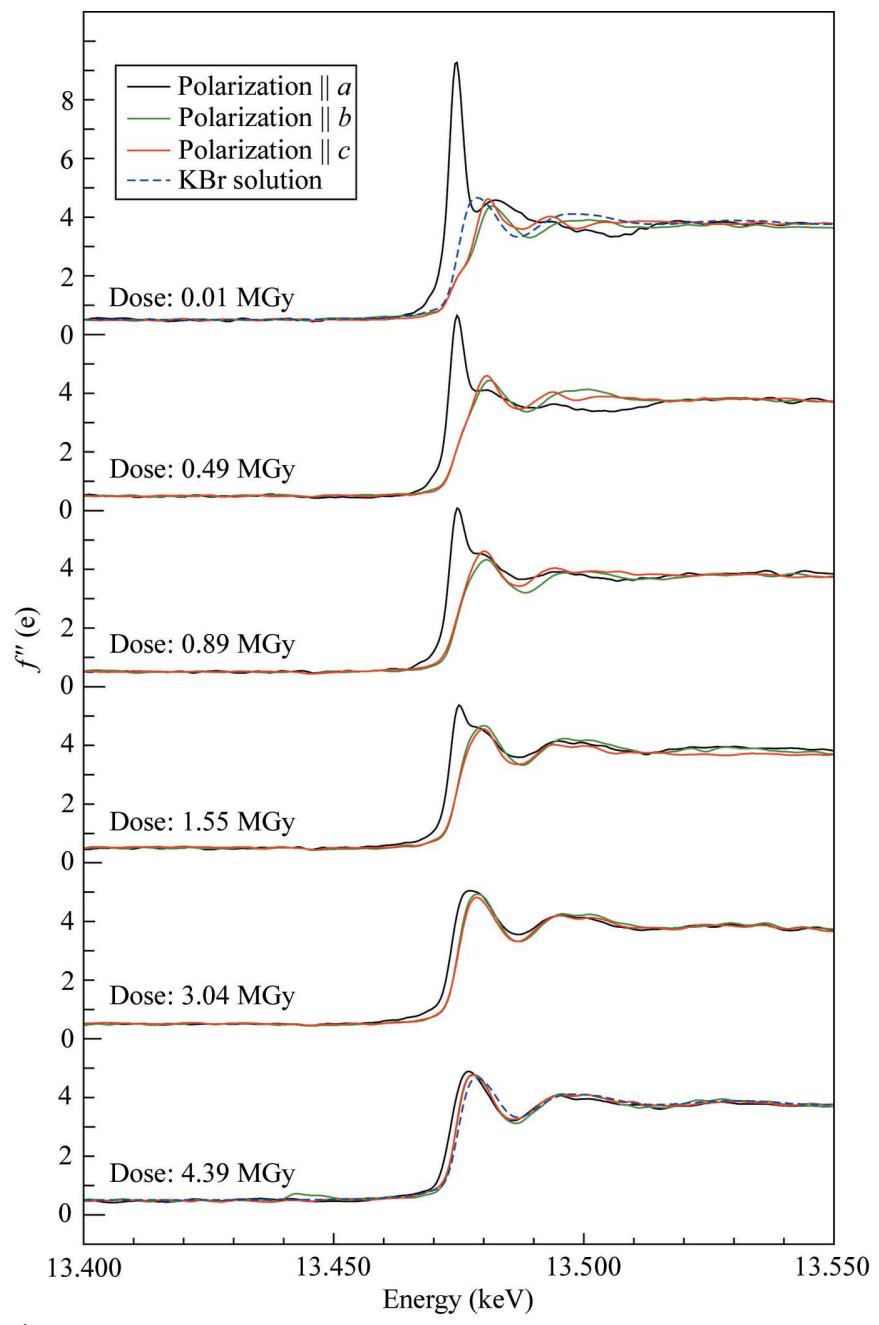

Figure 4

Polarized absorption spectra collected on a single crystal of DIS-BrU3 as a function of absorbed X-ray dose. The spectra were recorded for three different crystal orientations, in which the polarization direction of the $\mathrm{X}$-ray beam was respectively aligned with the crystal axes $a, b$ and $c$ (coloured in black, green and red, respectively). These crystal axes very nearly correspond to molecular directions defined as parallel to the $\mathrm{C}-\mathrm{Br}$ bonds ( $a$ axis), perpendicular to the $\mathrm{C}-\mathrm{Br}$ bonds and to the aromatic ring system of the brominated nucleobases ( $b$ axis) and perpendicular to the $\mathrm{C}-\mathrm{Br}$ bonds and contained in the plane of the aromatic ring system of the nucleobases ( $c$ axis). In between measurements, the crystal was irradiated in order to induce debromination. All spectra are on the same scale ( $f^{\prime \prime}$ in number of electrons). For the first and final triplets of spectra, a reference spectrum collected on a $0.1 \mathrm{M} \mathrm{KBr}$ solution is also superimposed (dashed blue line). It is not exactly coincident with the last spectrum obtained with the polarization aligned with the $a$ parameter, since the corresponding dose (4.39 MGy) left 10$15 \%$ of bromine uncleaved. 
in McGeehan et al. (2007) on the one hand and in the present study on the other hand. However, large values (6.7 and 7.1 MGy) had also been obtained for the two $\mathrm{Br}$ atoms of crystals comparable to those used in the present study (Schiltz et al., 2004). Since these large values relied on very crude dose estimates from previous work (Ennifar et al., 2002), they might be viewed as unreliable in comparison with the more accurate ones (1.33 and 0.74 MGy) from the present work. However, we will see in the following that considerably different $D$ values can indeed be obtained from identical crystals.

\section{Comparison of the kinetics of cleavage from fluorescence spectra and from crystallographic data}

In order to compare the bromine occupancy derived from fluorescence spectra with values from crystallographic data, we have carried out a series of measurements in which diffraction data collections were inserted between the recording of two consecutive spectra. Overall, seven data sets were collected up to $1.95 \AA$ resolution, each corresponding to the same angular range $\left(120^{\circ}\right)$ of crystal rotation (see $\left.\$ 2\right)$. The resulting experimental structure-factor amplitudes $F_{i}$ and the anomalous differences $\Delta \mathrm{ano}_{i}$ were compared in a pairwise manner, i.e. the $i$ th data set $F_{i}$ (for $i=2, \ldots, 7$ ) was compared with the first data set $F_{1}$ (see Fig. $2 a$ for $i=2,5$ and 7) and, analogously, each $\Delta \mathrm{ano}_{i}$ was compared with $\Delta \mathrm{ano}_{1}$ (Fig. $2 b$ ). These graphs show a decrease of correlation from the first to the last pairwise comparison (from $99.9 \%$ to $96.8 \%$ for the experimental $F$ values and from $66.2 \%$ to $27.3 \%$ for the $\Delta$ ano values). At first sight, one might think that this essentially reflects a strong deleterious effect of irradiation on the overall quality of the data. This is not exact, however, since a modest increase from $3.8 \%$ to $5.2 \%$ of the internal $R_{\text {merge }}$ on intensities (that is, from $1.9 \%$ to $2.6 \%$ on amplitudes) is observed from the first to the seventh data set, whereas the pairwise $R$ factors on amplitudes show a much more important increase $(3.6 \%, 4.8 \%, 6.3 \%, 8.6 \%, 14.7 \%$ and $28.2 \%)$ from $F_{1}$ versus $F_{2}$ to $F_{1}$ versus $F_{7}$. Moreover, the inflation is also apparent on the analogous scattergrams obtained with calculated data from models that differ only by a continuous decrease of the occupancy of the two $\mathrm{Br}$ atoms (Fig. $2 a$; see legend for the occupancy values). The corresponding pairwise $R$ factors are $1.9 \%, 3.3 \%, 4.6 \%, 6.4 \%, 7.8 \%$ and $11.4 \%$. The latter $R$ factors are smaller than the former because they are neither affected by the variance arising from experimental errors nor by the variance arising from damage other than bromine cleavage. However, the increase in $R$ factor from $1.9 \%$ to $6.4 \%$ for the first four calculated terms closely follows the increase from $3.6 \%$ to $8.6 \%$ for the first four experimental terms. This implies, as already mentioned (Ennifar et al., 2002), that the crystal quality was very little affected and that essentially the 'fingerprint' of X-rays was a rather 'clean' bromine cleavage, at least in this dose range, i.e. up to 5-6 MGy (see Fig. 3 for dose values). In contrast, the last two $R$-factor values from calculated data $(7.8 \%$ and $11.4 \%)$ increase less rapidly than their experimental counterparts $(14.7 \%$ and $28.2 \%)$, which means that beyond 5-6 MGy (in this particular case), damage other than bromine cleavage became the major source of variance between successive data sets. This is in agreement with the evolution of the $B$ factor obtained from Wilson plots (Wilson, 1949) between $2.8 \AA$ and the highest resolution $1.95 \AA$ (the lower resolution limit $2.8 \AA$ yields a good linearity of the plots; not shown). The successive values are $B_{1}=32.6$, $B_{2}=32.7, B_{3}=33.3, B_{4}=34.3, B_{5}=34.7, B_{6}=36.8$ and $B_{7}=42.8 \AA^{2}$, which highlights either a negligible or a weak loss of diffraction up to the fifth data set and a significant subsequent increase.

The decrease in correlation of the anomalous data (from $66 \%$ for $\Delta \mathrm{ano}_{1}$ versus $\Delta \mathrm{ano}_{2}$ to $27 \%$ for $\Delta \mathrm{ano}_{1}$ versus $\Delta \mathrm{ano}_{7}$; Fig. $2 b$ ) should also reflect the bromine cleavage. We have thus computed seven anomalous difference maps with the different sets of coefficients $\Delta \mathrm{ano}_{i}$ but with the same set of phases and we have compared the occupancies derived from the peak heights at the bromine positions ${ }^{2}$ with the occupancies derived from the absorption spectra measured between two data collections (Fig. 3). Notably, the occupancy curves derived from the anomalous data were very little dependent both on the resolution limits used for the calculations and on whether or not the $\mathrm{Br}$ atoms were included in the computation of phases. The reasonable agreement between the two independent estimates (Fig. 3) confirms that the occupancy derived from the absorption spectra is valuable. The $D$ values obtained from these different methods on the same crystal are $9.2 \pm 0.3$ and $11.8 \pm 0.5 \mathrm{MGy}$ for the two bromine sites from anomalous data and an 'averaged value' of $12.4 \pm 0.5 \mathrm{MGy}$ from the fluorescence spectra.

Two points are worth mentioning. Firstly, the occupancy values derived from the anomalous data appear to be slightly lower on average than those from the fluorescence spectra (Fig. 3). This might be the result of experimental noise affecting the anomalous data. Other examples are needed to decide whether or not this is real. Secondly, there exists a slight, but significant, difference between the $D$ value for each bromine site: $9.2 \pm 0.3$ and $11.8 \pm 0.5 \mathrm{MGy}$ from the red and blue lines, respectively, in Fig. 3. This is in agreement with the difference previously observed for the same bromine sites: 6.7 and 7.1 MGy (Schiltz et al., 2004). At variance with the present case, a rather large difference was reported for the $D$ values of two Br atoms in a DNA crystal: 2 and 4.5 MGy (McGeehan et al., 2007).

As indicated before, one can now compare the $D$ values obtained from different specimens of the same crystal form (namely, the crystal form without lividomycin that was used at the SLS). Our values (9.2 and 11.8 MGy from anomalous data and 12.4 MGy from fluorescence spectra), when compared with the value of $1.3 \mathrm{MGy}$ from fluorescence spectra alone, confirm that quite related crystals can yield quite different $D$ values. The same feature was also apparent in McGeehan et al.

\footnotetext{
2 To obtain occupancies from the peak heights, we took into account that the highest peak obtained with the coefficients $\Delta \mathrm{ano}_{1}$ was representative of slightly less than unit occupancy (since bromine cleavage started during the recording of the first fluorescence spectrum preceding the collection of the $F_{1}$ data set and progressed during that data collection). The corrected maximum occupancy was 0.96 instead of 1 .
} 


\section{research papers}

(2007), where a pair of crystals obtained under exactly the same conditions yielded $D$ values of 7.1 and $3.1 \mathrm{MGy}$. One can thus conclude that fluorescence spectra and crystallographic data yield consistent $D$ values on a given crystal, but that different specimens of the same crystal form may yield surprisingly different $D$ values. We will return to this point in light of the following results.

\section{Monitoring the effect of crystal orientation versus the beam polarization}

It is not uncommon to observe variations in the absorption spectra recorded on different crystals of the same sample, especially in the vicinity of an absorption edge. Quite often, these effects may be traced back to having arisen from different orientations of the crystals, implying different orientations of the covalent bonds involving the anomalously scattering atoms with respect to the polarization direction of the X-ray beam. The anomalous scattering factors for isolated atoms are spherically symmetric, but chemical bonding generally lowers the symmetry and induces a directional dependence of $f^{\prime}$ and $f^{\prime \prime}$ on the direction of linear polarization of the X-ray beam. This anisotropy of anomalous scattering is only present in the near-edge region and has been demonstrated experimentally in numerous investigations on salt and small-molecule compounds (Hodeau et al., 2001) as well as on selenated proteins (Bricogne et al., 2005).

It is thus important to distinguish between those modulations in the $\mathrm{Br} K$-edge absorption spectra that arise from $\mathrm{X}$-ray-induced radiolysis and those that arise from anisotropy of anomalous scattering. In order to make a clear comparison of both effects, we have recorded a series of polarized absorption spectra on an oriented single crystal of DIS-BrU3 as a function of X-ray irradiation. The position of the brominated uridine within each hairpin and the orientation of each loop-loop complex are such that the two $\mathrm{C}-\mathrm{Br}$ bonds in the asymmetric unit are almost parallel to each other and aligned (within $13^{\circ}$ ) with the crystal $a$ axis. Furthermore, since the crystals belong to the orthorhombic space group $C 222_{1}$, all symmetry-related $\mathrm{C}-\mathrm{Br}$ bonds are also almost aligned with the crystal $a$ axis. Also, the directions which are perpendicular to the planes of the aromatic ring systems of the brominated nucleobases are aligned (within $14^{\circ}$ ) with the crystal $b$ axis. In summary, the crystal $a, b$ and $c$ axes are very nearly aligned with the principal molecular directions (see Bricogne et al., 2005) at the level of the brominated nucleobases. Using a multi-axis goniometer, polarized X-ray absorption spectra were collected by successively aligning the crystal $a, b$ and $c$ axes with the polarization direction of the X-ray beam. The crystal was then irradiated in order to induce radiolysis of the $\mathrm{C}-\mathrm{Br}$ bonds and the recording of the three polarized absorption spectra was repeated at regular time intervals. The results of these measurements are shown in Fig. 4. As found previously (Bricogne et al., 2005), there is a strong anisotropy in the $f^{\prime \prime}$ spectrum of the DIS-BrU3 crystals and the intensity of the white line directly depends on the extent of alignment of the $\mathrm{C}-\mathrm{Br}$ bonds with the direction of $\mathrm{X}$-ray polarization. The anisotropy of the absorption spectra gradually disappears as the sample is irradiated. Anisotropy of anomalous scattering is the result of chemical bonding of the absorbing atoms. As radiolysis of the $\mathrm{C}-\mathrm{Br}$ bonds in the crystal progresses, the three polarized spectra become virtually identical. Moreover, after extensive irradiation, all three spectra very nearly correspond to the spectral signature of solvated $\mathrm{Br}^{-}$ions. It should be noted that all three of the polarized absorption spectra change as a result of X-ray irradiation. Whereas the $\mathrm{X}$-ray-induced changes are most prominent for the orientation which displays the white-line feature (i.e. when the $\mathrm{C}-\mathrm{Br}$ bonds are parallel to the polarization direction), a small but significant edge shift of about $3 \mathrm{eV}$ towards lower X-ray energies is observed for the other two crystal orientations. Thus, depending on the sample orientation, X-ray irradiation may induce edge shifts either towards higher or towards lower energies. These data fully agree with the results of Bricogne $e t$ al. (2005) that showed that both crystal orientation and X-ray irradiation can strongly modulate X-ray absorption spectra. Radiation-induced changes can be followed by X-ray absorption spectra, provided that all spectra are recorded on samples in the same orientation, since important variability of the spectra may merely result from a different orientation. Obviously, such effects were particularly visible on the crystals used for this study since the two $\mathrm{C}-\mathrm{Br}$ bonds (and their symmetry equivalents) were almost parallel. In a typical MAD experiment with a protein containing several selenomethionines, the fluorescence spectra would be an average of most likely unrelated $\mathrm{C}-$ Se bond orientations and therefore would not show up such dramatic differences.

Lastly, we would like to comment on the differences that were observed in the kinetics of $\mathrm{C}-\mathrm{Br}$ bond cleavage for identical crystals (and even for two $\mathrm{C}-\mathrm{Br}$ bonds in a given crystal). It might be thought that errors in X-ray dose quantification would account for the differences between identical crystals. However, both our own work with quantification relying on crystallography-based and fluorescence-based methods, as well as an independent study relying on Ramanbased methods (McGeehan et al., 2007), have shown significantly different $D$ values, which adds weight to the observations. Also, as already mentioned in $\S 1$, the 'obvious' explanation of a difference in solvent accessibility has too many exceptions to be retained as the unique explanation of differences within a given crystal. We thus hypothesized that the orientation of the cleavable bond relative to the beam polarization could not only influence the white-line feature, but also the kinetics of cleavage. More precisely, the cleavage efficiency would be maximum when a covalent bond is parallel to the beam polarization and minimum when it is perpendicular. Such a hypothesis was in agreement with the observation of the lowest $D$ value (0.74 MGy), obtained precisely with a crystal oriented for aligning the $\mathrm{C}-\mathrm{Br}$ bonds with the polarization vector (Fig. 4). We thus performed measurements of the kinetics of cleavage on identical crystals in different orientations. No significant variations could be detected (not shown), which disproved the hypothesis. Whether variations of the electric field, as hypothesized in Holton (2007), might 
explain the different $D$ values within a given crystal remains to be examined. In the particular case of the crystals used in this study, explicit calculation of the electric field within a unit cell would indeed take into consideration the possible influence of the slight variation in sequence and of the ligand present in one crystal form and absent in the other (see $\$ 2$ ).

\section{Discussion and conclusions}

Our results on bromine fully confirm those obtained on selenium by Holton (2007) that showed that it is possible to monitor the extent of specific radiation damage to anomalously scattering atoms by X-ray absorption spectra. Since the recording of X-ray absorption spectra in fluorescence mode is nowadays straightforward on most MAD synchrotron beamlines, performing such measurements at regular time intervals during an X-ray data collection is an easy way to detect the onset of specific radiation damage, thus giving the experimenter an opportunity to make appropriate decisions (e.g. change the sample or continue the data collection in view of exploiting RIP). The bottom line should be to record a spectrum at the beginning of the data collection (which is usually performed anyway) and a second one at the end of the data collection. Importantly, the two spectra should be measured in the same crystal orientation. A simple comparison of these two spectra will reveal whether or not the anomalously scattering atoms have suffered from radiation damage. In the former case, the success of SAD/MAD phasing may be compromised and the data-collection strategy may have to be modified. Alternatively, one could attempt to exploit the site-specific radiation damage for RIP. On the other hand, if no changes are discernable in the absorption spectra, radiation damage to the anomalously scattering atoms can be excluded and there would be no point in attempting RIP on such data.

In practice, in view of using the RIP method, it may be suggested to measure, in addition of the first 'zero-dose' spectrum, at least one other spectrum after some images have been collected (around 15 images). If an 'infinite-dose' spectrum is independently available (e.g. that from $\mathrm{NaBr}$ in solution or from a sufficiently 'burnt' crystal), (1) provides an estimate of the cleavage. Two points should be noted. Firstly, a short scan in energy around the peak would be sufficient to be effective (see Fig. 1); secondly, the implementation of an automatic procedure for such a measurement does not appear to be a difficult task. Obtaining an estimate of the cleavage could be of interest to tune the beam intensity in order to reach a predetermined fraction of cleavage (say $80 \%$ or $90 \%$ ) at the end of data collection. There certainly exists a joint optimum for the two parameters, beam intensity and total number of images, the latter being strongly dependent on the crystal symmetry to ensure redundancy. An implementation of these ideas in a program such as STRATEGY (Ravelli et al., 1997 ) would be of great interest. Importantly, our results as well as others show that each anomalous scatterer susceptible to cleavage needs an individual refinable $D$ value for the RIP method. It should also be mentioned that the same volume of the crystal should be irradiated during data collection, which may require defocusing the beam, as was performed in the present study. Indeed, if different parts of a crystal were irradiated variably (Schulze-Briese et al., 2005), it would not be possible to consider each individual intensity measurement to correspond to a given dose and thus to a given state of radiation damage.

A potential problem arises if iodine is to be used, since the wavelengths of its $K$ edge $(\lambda=0.374 \AA)$ and of its $L_{\mathrm{I}}$ edge $(\lambda=2.39 \AA)$ are not commonly accessible on synchrotron MAD beamlines. However, there are reports of successful data collection and phasing at very short wavelengths (for example, $\lambda=0.358 \AA$ at the Xe $K$ edge; Schiltz et al., 1997; see also Gonzalez et al., 1994). Such short wavelengths are obviously a problem for spot separation with large unit-cell parameters, but this inconvenience is mitigated because short wavelengths permit the use of longer crystal-to-detector distances owing to reduced air absorption and scattering.

\section{References}

Ambert, N., Vanwinsberghe, J. \& Dumas, P. (2006). CCP4 Newsl. 44, 4. Bernacchi, S., Ennifar, E., Toth, K., Walter, P., Langowski, J. \& Dumas, P. (2005). J. Biol. Chem. 280, 40112-40121.

Birkedal, H. (2000). PhD thesis. Université de Lausanne, Switzerland. Bressler, C., Saes, M., Chergui, M., Grolimund, M., Abela, R. \& Pattison, P. (2002). J. Chem. Phys. 116, 2955-2966.

Bricogne, G., Capelli, S. C., Evans, G., Mitschler, A., Pattison, P., Roversi, P. \& Schiltz, M. (2005). J. Appl. Cryst. 38, 168-182.

Burmeister, W. P. (2000). Acta Cryst. D56, 328-341.

Douzou, P., Hoa, G. H. \& Petsko, G. A. (1975). J. Mol. Biol. 96, 367-380.

Ennifar, E., Carpentier, P., Ferrer, J.-L., Walter, P. \& Dumas, P. (2002). Acta Cryst. D58, 1262-1268.

Ennifar, E. \& Dumas, P. (2006). J. Mol. Biol. 356, 771-782.

Ennifar, E., Meyer, J. E., Buchholz, F., Stewart, A. F. \& Suck, D. (2003). Nucleic Acids Res. 31, 5449-5460.

Ennifar, E., Paillart, J. C., Bodlenner, A., Walter, P., Weibel, J. M., Aubertin, A. M., Pale, P., Dumas, P. \& Marquet, R. (2006). Nucleic Acids Res. 34, 2328-2339.

Ennifar, E., Walter, P., Ehresmann, B., Ehresmann, C. \& Dumas, P. (2001). Nature Struct. Biol. 8, 1064-1068.

Evans, G. \& Pettifer, R. F. (2001). J. Appl. Cryst. 34, 82-86.

Evans, G., Polentarutti, M., Djinovic Carugo, K. \& Bricogne, G. (2003). Acta Cryst. D59, 1429-1434.

Fioravanti, E., Vellieux, F. M., Amara, P., Madern, D. \& Weik, M. (2007). J. Synchrotron Rad. 14, 84-91.

Gonzalez, A., Denny, R. \& Nave, C. (1994). Acta Cryst. D50, 276-282.

Gonzalez, A. \& Nave, C. (1994). Acta Cryst. D50, 874-877.

Haas, D. J. \& Rossmann, M. G. (1970). Acta Cryst. B26, 998-1004.

Henderson, R. (1990). Proc. R. Soc. Lond. B, Biol. Sci. 241, 6-8.

Hodeau, J. L., Favre-Nicolin, V., Bos, S., Renevier, H., Lorenzo, E. \&

Berar, J. F. (2001). Chem. Rev. 101, 1843-1867.

Holton, J. M. (2007). J. Synchrotron Rad. 14, 51-72.

Hope, H. (1988). Acta Cryst. B44, 22-26.

Hunter, D. B., Bertsch, P. M., Kemner, K. M. \& Clark, S. B. (1997). J. Phys. IV , 7, C2-767-C2-771.

McGeehan, J. E., Carpentier, P., Royant, A., Bourgeois, D. \& Ravelli, R. B. G. (2007). J. Synchrotron Rad. 14, 99-108.

Murray, J. W., Garman, E. F. \& Ravelli, R. B. G. (2004). J. Appl. Cryst. 37, 513-522.

Otwinowski, Z. \& Minor, W. (1997). Methods Enzymol. 276, 307-326. 
Owen, R. L., Rudino-Pinera, E. \& Garman, E. F. (2006). Proc. Natl Acad. Sci. USA, 103, 4912-4917.

Petsko, G. A. (1975). J. Mol. Biol. 96, 381-392.

Ramagopal, U. A., Dauter, Z., Thirumuruhan, R., Fedorov, E. \& Almo, S. C. (2005). Acta Cryst. D61, 1289-1298.

Ravelli, R. B. \& Garman, E. F. (2006). Curr. Opin. Struct. Biol. 16, 624-629.

Ravelli, R. G. B., Leiros, H.-K. S., Pan, B., Caffrey, M. \& McSweeney, S. (2003). Structure Fold. Des. 11, 217-224.

Ravelli, R. B. \& McSweeney, S. M. (2000). Structure Fold. Des. 8, 315-328.

Ravelli, R. B. G., Nanao, M. H., Lovering, A., White, S. \& McSweeney, S. (2005). J. Synchrotron Rad. 12, 276-284.

Ravelli, R. B. G., Sweet, R. M., Skinner, J. M., Duisenberg, A. J. M. \& Kroon, J. (1997). J. Appl. Cryst. 30, 551-554.

Schiltz, M., Dumas, P., Ennifar, E., Flensburg, C., Paciorek, W., Vonrhein, C. \& Bricogne, G. (2004). Acta Cryst. D60, 1024-1031.

Schiltz, M., Kvick, A., Svensson, O. S., Shepard, W., de La Fortelle, E., Prange, T., Kahn, R., Bricogne, G. \& Fourme, R. (1997). J. Synchrotron Rad. 4, 287-297.
Schulze-Briese, C., Wagner, A., Tomizaki, T. \& Oetiker, M. (2005). J. Synchrotron Rad. 12, 261-267.

Smith, J. L., Hendrickson, W. A., Terwilliger, T. C. \& Berendzen, J. (2001). International Tables for Crystallography, Vol. F, edited by M. G. Rossmann \& E. Arnold, pp. 299-309. Dordrecht: Kluwer Academic Publishers.

Teng, T. \& Moffat, K. (2000). J. Synchrotron Rad. 7, 313317.

Weik, M., Ravelli, R. B., Kryger, G., McSweeney, S., Raves, M. L., Harel, M., Gros, P., Silman, I., Kroon, J. \& Sussman, J. L. (2000). Proc. Natl Acad. Sci. USA, 97, 623-628.

Wilson, A. J. C. (1949). Acta Cryst. 2, 318-321.

Xie, J., Wang, L., Wu, N., Brock, A., Spraggon, G. \& Schultz, P. G. (2004). Nature Struct. Biol. 22, 1297-1301.

Yano, J., Kern, J., Irrgang, K. D., Latimer, M. J., Bergmann, U., Glatzel, P., Pushkar, Y., Biesiadka, J., Loll, B., Sauer, K., Messinger, J., Zouni, A. \& Yachandra, V. K. (2005). Proc. Natl Acad. Sci. USA, 102, 12047-12052.

Zwart, P. H., Banumathi, S., Dauter, M. \& Dauter, Z. (2004). Acta Cryst. D60, 1958-1963. 